\title{
Macrophage Migration Inhibitory Factor Is a Possible Candidate for the Induction of Microalbuminuria in Diabetic $d b / d b$ Mice
}

\author{
Tamaki Watanabe, ${ }^{a}$ Naoko H. Tomioka, ${ }^{b}$ Masaru Doshi, ${ }^{b}$ Shigekazu Watanabe,${ }^{a}$ \\ Masao Tsuchiya, ${ }^{a}$ and Makoto Hosoyamada*, \\ ${ }^{a}$ Practical Pharmacy, Faculty of Pharma-Sciences, Teikyo University; and ${ }^{b}$ Human Physiology and Pathology, \\ Faculty of Pharma-Sciences, Teikyo University; 2-11-1 Kaga, Itabashi-ku, Tokyo 173-8605, Japan. \\ Received August 21, 2012; accepted February 19, 2013
}

Preventing the onset of microalbuminuria in diabetic nephropathy is a problem that needs urgent rectification. The use of a mouse model for diabetes is vital in this regard. For example, $d b / d b$ mice exhibit defects in the leptin receptor Ob-Rb sub-type, while the ob/ob strain exhibits defects in the leptin ligand. These mouse strains demonstrate type 2 diabetes, either with or without microalbuminuria, respectively. The purpose of the present study was to use DNA microarray technology to screen for the gene responsible for the onset of diabetic microalbuminuria. Using Affymetrix Mouse Gene ST 1.0 arrays, microarray analysis was performed using total RNA from the kidneys of $o b$ control, $o b / o b, d b / m$, and $d b / d b$ mice. Microarray and quantitative reverse transcription-polymerase chain reaction (RT-PCR) indicated that transcription of the macrophage migration inhibitory factor (MIF) gene was significantly enhanced in the kidneys of $d b / d b$ mice. Western blotting showed that levels of MIF protein was enhanced in the kidneys of both diabetic $d b / d b$ and $o b / o b$ mice. On the other hand, elevation of urinary MIF excretion detected by enzyme-linked immunosorbent assay (ELISA) was only in $d b / d b$ mice and preceded the onset of microalbuminuria. Immunofluorescence studies revealed that MIF was expressed in mouse kidney glomeruli. While MIF expression was enhanced in the diabetic kidneys of both mouse strains, the elevated secretion from $d b / d b$ mouse kidneys may be responsible for initiating the onset of microalbuminuria in diabetic nephropathy.

Key words macrophage migration inhibitory factor; diabetic nephropathy; microalbuminuria; $d b / d b$ mouse; $o b / o b$ mouse

Diabetic nephropathy is the primary causative disease leading to dialysis in Japan. ${ }^{1)}$ The cumulative incidence of nephropathy after 30 years of post-pubertal diabetes was reported to be significantly higher in type 2 diabetic patients $(44.4 \%)$ than in type 1 diabetic patients $(20.2 \%){ }^{2)}$ However, diabetic nephropathy does not occur in all patients with type 2 diabetes mellitus, implying that there might be an unknown underlying mechanism to prevent the onset of diabetic nephropathy. Establishing the identity of such a mechanism is of vital importance.

It is thought that the onset of microalbuminuria, considered to be a marker of early diabetic nephropathy, must be suppressed in order to inhibit the onset and aggravation of nephropathy in type 2 diabetes. Two murine strains are available as an animal model for type 2 diabetes: $d b / d b$ mice in which microalbuminuria develops at 8 weeks of age ${ }^{3)}$; and $o b / o b$ mice that do not develop microalbuminuria at all. It is thought that a gene cluster associated with the onset of microalbuminuria could therefore be identified by comparing differences in gene expression between these two mouse strains, without considering a specific disease-developing mechanism underlying microalbuminuria.

The purpose of this study was therefore to identify a gene associated with the onset of microalbuminuria in the type 2 diabetic model mouse using a DNA microarray approach. Macrophage migration inhibitory factor (MIF) was identified as a gene for which expression changed in $d b / d b$ mice, but not in $o b / o b$ mice. Since earlier reports indicate that MIF is associated with glomerulonephritis and proteinuria, ${ }^{4)}$ the present study also aimed to analyze the expression of MIF in the

The authors declare no conflict of interest. kidneys of $d b / d b$ and $o b / o b$ mice.

\section{MATERIALS AND METHODS}

Animals Experiments involved male $d b / d b$ and $o b / o b$ mice and non-diabetic controls $(d b / m$ and $o b$ control mice) that were 2 months of age. Urine was sampled using metabolic cages. Mice were anesthetized by intra-peritoneal injections of $50 \mathrm{mg} / \mathrm{kg}$ pentobarbital in order to obtain blood and kidney samples. Serum glucose was measured enzymatically using Glucose CII-test Wako (Wako Pure Chemical Industries, Ltd., Osaka, Japan). Serum insulin and leptin was assayed using Mouse Insulin enzyme-linked immunosorbent assay (ELISA) kit (H-type) and Mouse Leptin ELISA kit, respectively (Shibayagi, Gunma, Japan). Urinary albumin was assayed using Albuwell M kit (Exocell Inc., Philadelphia, U.S.A.). Macrophage Migration Inhibitory Factor in plasma or urine was assayed by ELISA kit for mouse MIF (Uscn Life Science Inc., Houston, U.S.A.). Serum and urinary creatinine was assayed using HPLC as described previously. ${ }^{5)}$ All animal experiments were carried out in accordance with Teikyo University Guide for the Care and Use of Laboratory Animals.

Microarray Analysis Total RNA was extracted from the kidneys of $o b$ control, ob/ob, $d b / m$, and $d b / d b$ mice and purified as described previously. ${ }^{6}$ Microarray analysis was performed using Affymetrix Mouse Gene ST 1.0 arrays and analyzed using Expression Console (PharmaFrontier Co., Ltd., Kyoto, Japan).

Real-Time Polymerase Chain Reaction (PCR) cDNA was prepared from total RNA using a High-Capacity cDNA Reverse Transcription Kit. Real-time PCR analyses were subsequently conducted as described previously $\left.{ }^{6}\right)$ using TaqMan 
probes corresponding to macrophage migration inhibitory factor (Mm01611157_gH), and 18s ribosomal RNA (rRNA) as an internal standard.

Western Blotting Kidneys were homogenized in a specific solution $(20 \mathrm{~mm}$ Tris, $3 \mathrm{~m}$ Urea, $0.5 \%$ CHAPS, $0.1 \mathrm{~m}$ $\mathrm{NaCl}, \mathrm{pH}$ 7.4) containing protease inhibitors (Complete Mini, Roche Diagnostics Japan, Tokyo). Homogenates ( $5 \mu \mathrm{g} / \mathrm{lane})$ were separated by sodium dodecyl sulfate-polyacrylamide gel electrophoresis (SDS-PAGE) and transferred to a Nitrocellulose membrane. After blocking, membranes were stained with anti-mouse MIF antibody $(0.5 \mu \mathrm{g} / \mathrm{mL}$; FL-115 (sc-20121); Santa Cruz Biotechnology, Inc., Santa Cruz, CA, U.S.A.) and horseradish peroxidase (HRP) conjugated anti-rabbit immunoglobulin $\mathrm{G}$ (IgG) antibody $(1: 10000$, ZYM 65-6120, Life Technologies Japan Ltd., Tokyo). An HRP-conjugated anti- $\beta$ actin antibody $(1: 40000, \beta$ actin $C 4$, sc-47778, Santa Cruz Biotechnology, Inc.) was used to calibrate sample loading.
Products were detected with an ECL kit (GE Healthcare UK Ltd., Little Chalfont, U.K.).

Immunofluorescence Paraffin sections were prepared from the kidneys of $o b$ control, ob/ob, $d b / m$, and $d b / d b$ mice which had been perfused with $4 \%$ paraformaldehyde in $30 \mathrm{~mm} N$-(2-hydroxyethyl)piperazine- $N$-2-ethanesulfonic acid (HEPES) buffer. Deparaffinized sections were stained with anti-mouse MIF antibody (1:50, Santa Cruz Biotechnology, Inc.) and Cy5-conjugated anti-rabbit $\operatorname{IgG}(\mathrm{H}+\mathrm{L})(1: 100$, A10523, Life Technologies Japan Ltd., Tokyo, Japan). ${ }^{7)}$

Statistical Analyses All data, except that arising from DNA microarrays, are expressed as mean \pm standard error (S.E.M.). Differences between groups were analyzed statistically using the student's $t$-test. $p$ values $<0.05$ were considered statistically significant.

Table 1. Comparison of Clinical State of Diabetes

\begin{tabular}{lcccc}
\hline \hline & $d m / m$ & $d b / d b$ & $o b$ control & $o b / o b$ \\
\hline Body weight $(\mathrm{g})$ & $24.2 \pm 0.5$ & $41.1 \pm 0.9^{* *}$ & $27.5 \pm 0.4$ & $45.9 \pm 0.7^{* *}$ \\
Volume of urine $(\mathrm{mL} / \mathrm{d})$ & $1.0 \pm 0.1$ & $11.9 \pm 1.3^{* *}$ & $1.3 \pm 0.2$ & $6.5 \pm 0.8^{* *}$ \\
Serum glucose $(\mathrm{mg} / \mathrm{dL})$ & $214 \pm 11$ & $615 \pm 52^{* *}$ & $308 \pm 21$ & $366 \pm 28$ \\
Serum insulin $(\mathrm{ng} / \mathrm{mL})$ & $2.3 \pm 0.6$ & $11.5 \pm 3.3$ & $0.9 \pm 0.3$ & $30.6 \pm 6.3^{*}$ \\
Creatinine clearance $\left(\mathrm{mL} / \mathrm{d} / \mathrm{cm}^{2}\right)$ & $0.23 \pm 0.04$ & $0.53 \pm 0.05^{* *}$ & $0.26 \pm 0.05$ & $0.49 \pm 0.04^{* *}$ \\
Urinary albumin $(\mu \mathrm{g} / \mathrm{d})$ & $45.5 \pm 11.3$ & $332.4 \pm 79.9^{*, \#}$ & & $31.5 \pm 9.5$ \\
In-blood leptin $(\mathrm{pg} / \mathrm{mL})$ & $49 \pm 21$ & $7828 \pm 1232^{*, \#}$ & & n.d. \\
\hline
\end{tabular}

$* p<0.05, * * p<0.01$ vs. control, ${ }^{\#} p<0.01$ vs. ob/ob ( $n=4-10$, mean \pm S.E.M.).

A.

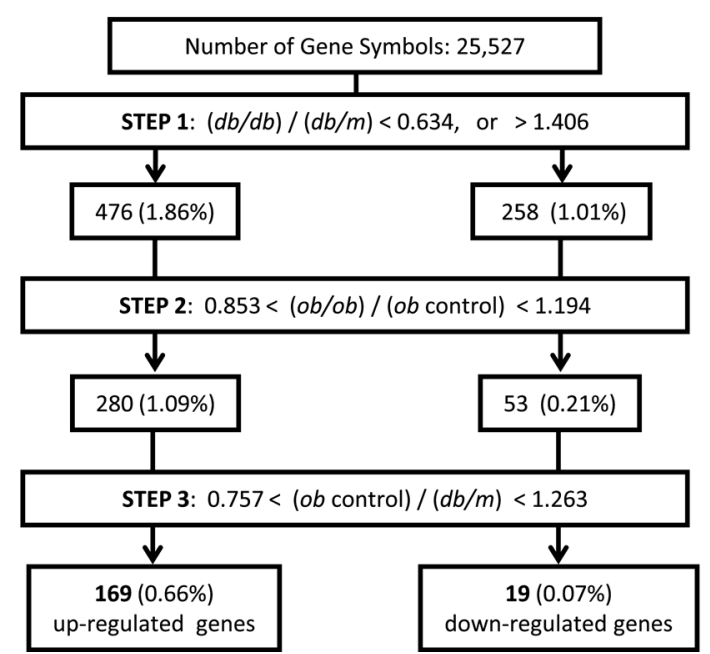

B.
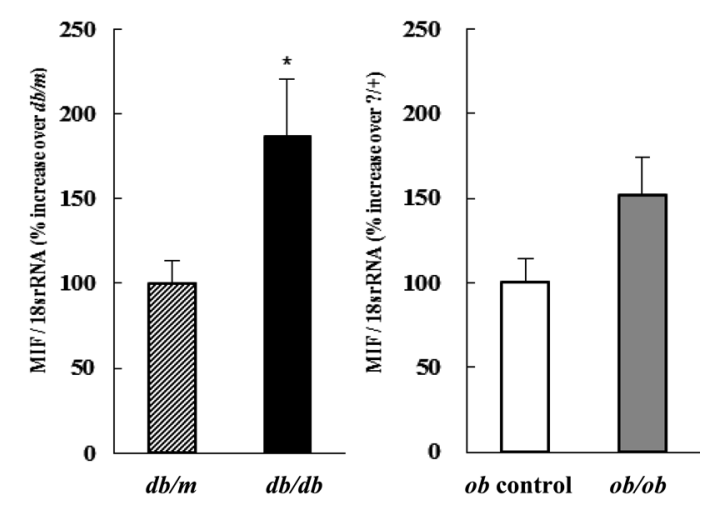

Fig. 1. Screening of Candidate Genes Underlying Microalbuminuria in Diabetic $d b / d b$ Mice

(A) Microarray study and data analysis. STEP1: Genes for which the expression ratio of $(d b / d b) /(d b / m)$ was higher than the mean +2 S.D. or lower than the mean -2 S.D. were screened. Since the gene expression ratio of $(\mathrm{db} / \mathrm{db}) /(\mathrm{db} / \mathrm{m})$ was $1.020 \pm 0.193$ (mean \pm S.D.) from a total of 25527 genes, 476 genes were induced by over 1.406 -fold and 258 genes were suppressed below 0.634 -fold. STEP 2: Genes for which the expression ratio of $(o b / o b) /(o b$ control) was higher than the mean +1 S.D. or lower than the mean-1S.D. were excluded as genes for which expression changed by diabetes. Since the expression ratio of $(\mathrm{ob} / \mathrm{ob}) /(\mathrm{ob}$ control) was $1.026 \pm 0.168$ from 25527 genes, 280 genes in 476 genes, and 53 genes in the 258 genes for which the expression ratio of $(o b / o b) /(o b$ control) was between 0.853- and 1.194-fold were screened. STEP 3: Genes for which the expression ratio of $(o b$ control $) /(d b / m)$ was higher than the mean +1 S.D. or lower than the mean $-1 \mathrm{~S}$.D. were excluded as genes for which expression changed by strain differences. The expression ratio for $(o b$ control $) /(d b / m)$ was $1.010 \pm 0.253$ from 25527 genes. Consequently, 169 genes of the 280 genes, and 19 genes of the 53 genes, for which the expression ratio of $(o b / o b) /(o b$ control) was between 0.757 - and 1.263-fold were screened as candidates of microalbuminuria-related genes. (B) Messenger RNA levels of MIF determined by quantitative PCR. ob control $v s$. $o b / o b$ and the $d b / m v s$. $d b / d b$ strains were compared. The amount of MIF transcription in the kidney was elevated in $d b / d b$ mice $(n=4-6$, mean \pm S.E.M., $* p<0.05)$. 


\section{RESULTS AND DISCUSSION}

Serum glucose levels of $d b / d b$ mice was significantly higher than those of $d b / m$ mice ( $p=0.017$ ), while those of $o b / o b$ mice tended to be higher than those of $o b$ control mice $(p=0.056)$. However, the urinary volume, serum insulin, and body weight of $d b / d b$ and $o b / o b$ mice were significantly higher than those of control mice. Therefore, type 2 diabetes-induced polyuria and obesity appears to have been demonstrated in both $d b / d b$ and $o b / o b$ mice. The obesity of $o b / o b$ mice was caused by defective leptin, which normally suppresses over-eating by stimulating the satiety center in the hypothalamus. Obesity in $d b / d b$ mice is known to be caused by defects in leptin signaling at the satiety center ${ }^{8)}$ (Table 1). Microalbuminuria were clearly observed in $d b / d b$ mice $(332.4 \pm 79.9 \mu \mathrm{g} / \mathrm{d}, p<0.01 v s$. $o b / o b$ mice), however, neither $d b / m$ mice $(45.5 \pm 11.3 \mu \mathrm{g} / \mathrm{d})$ nor $o b / o b$ mice $(31.5 \pm 9.5 \mu \mathrm{g} / \mathrm{d})$ were free from microalbuminuria (Table 1). This concurs with an earlier report ${ }^{3)}$ which reported that all $d b / d b$ mice develop microalbuminuria at 8 weeks of age, while $o b / o b$ mice did not develop microalbuminuria at all. Therefore, these two strains of diabetic mice are appropriate models for DNA microarray analysis in an attempt to identify microalbuminuria-related genes.

Creatinine clearance of $d b / d b \quad\left(0.53 \pm 0.05 \mathrm{~mL} / \mathrm{d} / \mathrm{cm}^{2}\right)$ and $o b / o b \quad\left(0.49 \pm 0.04 \mathrm{~mL} / \mathrm{d} / \mathrm{cm}^{2}\right)$ mice was significantly higher $(p=0.002,0.043)$ than those of $d b / m\left(0.23 \pm 0.04 \mathrm{~mL} / \mathrm{d} / \mathrm{cm}^{2}\right)$ and $o b$ control $\left(0.26 \pm 0.05 \mathrm{~mL} / \mathrm{d} / \mathrm{cm}^{2}\right)$ mice, respectively (Table 1). These results imply that microalbuminuria may not always be induced by glomerular hyper-filtration. Consequently, this indicates that there may be another candidate underlying the cause of microalbuminuria, other than glomerular hyperfiltration.

In order to screen candidates for microalbuminuria-related genes with $d b / d b$ and $o b / o b$ strains, we conducted three microarray data analysis steps (Fig. 1A). In STEP 1, up or down-regulated genes were determined as candidates for microalbuminuria-related genes. Genes for which the expression ratio of $(d b / d b) /(d b / m)$ was higher than (mean+2S.D.) or lower than (mean-2S.D.) were screened. In STEP 2, genes for which the expression ratio of $(o b / o b) /(o b$ control) was higher than the mean+1S.D. or lower than the mean-1S.D. were excluded as genes for which expression changed not by microalbuminuria, but by diabetes. In STEP 3, genes for which the expression ratio of ( $o b$ control) $/(d b / m)$ was higher than the mean+1S.D. or lower than the mean-1S.D. were excluded as genes for which expression changed not by microalbuminuria, but by differences involving the genetic background of $d b / d b$ and $o b / o b$ strains. Of the 25527 genes expressed in the mouse kidney, the expression of 169 and 19 genes were enhanced or suppressed as candidates for microalbuminuria-related genes in $d b / d b$ mouse kidney, respectively. Table 2 demonstrates the 169 up-regulated genes and the 19 down-regulated genes in the order of expression levels of $d b / d b$ mouse kidney.

Microarray studies demonstrated that macrophage migration inhibitory factor gene (MIF) was the most expressed gene in the 169 up-regulated microalbuminuria-related genes. Using quantitative PCR, MIF mRNA levels in $d b / d b$ mice were significantly higher than those in $d b / m$ mice (Fig. 1B). Moreover, the production of MIF is known to increase at the crescentic anti-glomerular basement membrane glomerulonephritis with nephrosis ${ }^{9}$ ) while proteinuria is known to occur in glomerular
Table 2. DNA Microarray Data

\begin{tabular}{|c|c|c|}
\hline Gene & Full name & Ratio \\
\hline \multicolumn{3}{|c|}{ Up-regulated genes } \\
\hline Gm16379 & macrophage migration inhibitory factor-like & 1.556 \\
\hline Mif & macrophage migration inhibitory factor & 1.625 \\
\hline Mif & macrophage migration inhibitory factor & 1.640 \\
\hline Nrldl & nuclear receptor subfamily 1 , gr. $\mathrm{D}, \mathrm{mbr} .1$ & 1.558 \\
\hline Aqp2 & aquaporin 2 & 1.626 \\
\hline Prodh & proline dehydrogenase & 1.732 \\
\hline Efhdl & EF hand domain containing 1 & 1.628 \\
\hline Scnnla & sodium channel, nonvoltage-gated 1 alpha & 1.447 \\
\hline Vamp2 & vesicle-associated membrane protein 2 & 1.651 \\
\hline Acot 2 & acyl-CoA thioesterase 2 & 1.527 \\
\hline Cyp2d9 & cytochrome P450, fam. 2d, polypeptide 9 & 1.469 \\
\hline Hspala & heat shock protein $1 \mathrm{~A}$ & 1.520 \\
\hline Tspan 7 & tetraspanin 7 & 1.464 \\
\hline Ifi30 & interferon gamma inducible protein 30 & 1.642 \\
\hline Pctk3 & PCTAIRE-motif protein kinase 3 & 1.478 \\
\hline Cabcl & chaperone, $\mathrm{ABC} 1$ activity of bc 1 complex like & 1.545 \\
\hline Mfge8 & milk fat globule-EGF factor 8 protein, tr-var. 1 & 1.416 \\
\hline Dmgdh & dimethylglycine dehydrogenase precursor & 1.523 \\
\hline Cyp $27 a 1$ & cytochrome P450, fam. $27 \mathrm{a}$, polypeptide 1 & 1.492 \\
\hline Paqr 5 & progestin and adipoQ receptor family mbr. V & 1.419 \\
\hline Aqp3 & aquaporin 3 & 1.467 \\
\hline$P d x k$ & pyridoxal kinase & 1.569 \\
\hline Dnajb4 & DnaJ (Hsp40) homolog, subfam. B, mbr. 4 & 1.914 \\
\hline Scnnlg & sodium channel, nonvoltage-gated 1 gamma & 1.421 \\
\hline Sec14l1 & SEC14-like 1 & 1.517 \\
\hline Hspala & heat shock protein $1 \mathrm{~A}$ & 2.004 \\
\hline Scnn $1 b$ & sodium channel, nonvoltage-gated 1 beta & 1.425 \\
\hline $\operatorname{Brd2}$ & bromodomain containing 2 , var. 2 & 1.467 \\
\hline Nicn 1 & nicolin 1 & 1.408 \\
\hline Gstt3 & glutathione $S$-transferase, theta 3 & 1.713 \\
\hline Gys1 & glycogen synthase 1 , muscle & 1.578 \\
\hline$H k 1$ & hexokinase 1 , var. 2 & 1.517 \\
\hline Abhd6 & abhydrolase domain containing 6 & 1.454 \\
\hline Gsta4 & glutathione $S$-transferase, alpha 4 & 1.551 \\
\hline Rnf186 & ring finger protein 186 & 1.835 \\
\hline Angptl3 & angiopoietin-like 3 & 1.890 \\
\hline Myof & myoferlin & 1.619 \\
\hline Foxil & forkhead box I1 & 1.696 \\
\hline Fam38a & Fam 38 a protein gene & 1.421 \\
\hline Fmo4 & flavin containing monooxygenase 4 & 1.491 \\
\hline Sfxn2 & sideroflexin 2 & 1.430 \\
\hline Bag3 & BCL2-associated athanogene 3 & 1.490 \\
\hline Slc14a1 & solute carrier fam. 14 (urea), mbr. 1 & 2.437 \\
\hline Fbxo44 & F-box protein 44 , tr-var. 1 & 1.442 \\
\hline Deaf1 & $\begin{array}{l}\text { deformed epidermal autoregulatory factor } 1 \text {, } \\
\text { tr-var. } 2\end{array}$ & 1.426 \\
\hline 4930572J05Rik & RIKEN cDNA & 1.638 \\
\hline Abpl & amiloride binding protein 1 , tr-var. 3 & 1.706 \\
\hline Wsbl & $\begin{array}{l}\text { WD repeat \& SOCS box-containing } 1 \text {, } \\
\text { tr-var. } 1\end{array}$ & 1.928 \\
\hline Gyltl1b & glycosyltransferase-like 1B & 1.572 \\
\hline Mpzl2 & myelin protein zero-like 2 & 1.605 \\
\hline Sulf2 & sulfatase 2 (Sulf2) & 1.422 \\
\hline Dnajbl & DnaJ (Hsp40) homolog, subfam. B, mbr. 1 & 1.413 \\
\hline Fus & fusion, from $\mathrm{t}(12 ; 16)$ malignant liposarcoma & 1.426 \\
\hline Htra2 & HtrA serine peptidase 2 & 1.425 \\
\hline Shmt1 & serine hydroxymethyltransferase 1 & 1.474 \\
\hline$A b t b 2$ & ankyrin repeat and BTB domain containing 2 & 1.488 \\
\hline Sycn & syncollin & 1.440 \\
\hline Slc $45 a 3$ & solute carrier family 45, mbr. 3 & 1.487 \\
\hline 2310081J21Rik & adult male tongue cDNA, RIKEN & 1.447 \\
\hline
\end{tabular}


Table 2. DNA Microarray Data

\begin{tabular}{|c|c|c|}
\hline 2310081J21Rik & adult male tongue cDNA, RIKEN & 1.447 \\
\hline 2310081J21Rik & adult male tongue cDNA, RIKEN & 1.447 \\
\hline Cyp $2 s 1$ & cytochrome P450, fam. 2s, polypeptide 1 & 1.421 \\
\hline Aldoc & aldolase $\mathrm{C}$, fructose-bisphosphate & 1.543 \\
\hline Mgll & monoglyceride lipase & 1.419 \\
\hline Unc45a & unc-45 homolog $\mathrm{A}$ & 1.425 \\
\hline$P f k p$ & phosphofructokinase, platelet & 1.503 \\
\hline Prkag3 & AMP kinase, gamma 3, non-catatlytic subunit & 1.790 \\
\hline Shpk & sedoheptulokinase & 1.496 \\
\hline D430041B17Rik & RIKEN cDNA & 1.425 \\
\hline Fstl3 & follistatin-like 3 & 1.463 \\
\hline Tubb2a & tubulin, beta $2 \mathrm{~A}$ & 1.411 \\
\hline Bcl6 & B-cell CLL/lymphoma 6 & 1.522 \\
\hline Upp2 & uridine phosphorylase 2 & 1.452 \\
\hline Pappa & pregnancy-associated plasma protein A & 1.532 \\
\hline Arrdc4 & arrestin domain containing 4 , tr-var. 1 & 1.503 \\
\hline $\operatorname{Ier} 5 l$ & immediate early response 5 -like & 1.452 \\
\hline Acot1 & acyl-CoA thioesterase 1 & 1.606 \\
\hline Ppm 1k & protein phosphatase $1 \mathrm{~K}$ & 1.452 \\
\hline $2610301 F 02 R i k$ & MCG142258, isoform CRA & 1.485 \\
\hline$A K 220484$ & cDNA sequence & 1.600 \\
\hline Atp $2 b 4$ & ATPase, $\mathrm{Ca}^{2+}$ transporting, plasma mbr. 4 & 1.616 \\
\hline Snrpg & small nuclear ribonucleoprotein polypeptide $\mathrm{G}$ & 1.450 \\
\hline Ddit4l & DNA-damage-inducible transcript 4-like & 1.507 \\
\hline Sntal & syntrophin, acidic 1 & 1.511 \\
\hline Affl & AF4/FMR2 fam., mbr. 1 & 1.407 \\
\hline Cuedcl & CUE domain containing 1 & 1.476 \\
\hline Rasl11b & RAS-like, fam. 11, mbr. B & 1.487 \\
\hline Gprc5b & $\begin{array}{l}\text { G protein-coupled receptor, fam. C, gr. } 5 \text {, } \\
\text { mbr. B }\end{array}$ & 1.423 \\
\hline Brms 1 & breast cancer metastasis-suppressor 1 & 1.415 \\
\hline Lypd6 & LY6/PLAUR domain containing 6 & 1.462 \\
\hline 1810010H24Rik & RIKEN cDNA & 1.550 \\
\hline$H s d 17 b 14$ & hydroxysteroid (17-beta) dehydrogenase 14 & 1.432 \\
\hline Tspan1 & tetraspanin 1 & 1.411 \\
\hline Fbp2 2 & fructose bisphosphatase 2 & 1.488 \\
\hline Th1l & TH1-like homolog & 1.465 \\
\hline Penk & preproenkephalin & 1.638 \\
\hline Gpt & glutamic pyruvic transaminase, soluble & 1.527 \\
\hline Freq & frequenin homolog & 1.513 \\
\hline $1110006024 R i k$ & RIKEN cDNA & 1.576 \\
\hline Cck & cholecystokinin & 1.407 \\
\hline Nes & nestin & 1.440 \\
\hline Dph2 & DPH2 homolog & 1.419 \\
\hline Aen & apoptosis enhancing nuclease, tr-var. 1 & 1.557 \\
\hline Krt34 & keratin 34 & 1.407 \\
\hline Fosb & FBJ osteosarcoma oncogene B & 1.599 \\
\hline Cadps 2 & $\mathrm{Ca}^{2+}$-dep. activator protein for secretion 2 & 1.482 \\
\hline Tmem54 & transmembrane protein 54 & 1.477 \\
\hline Nfil3 & nuclear factor, interleukin 3, regulated & 1.466 \\
\hline Spag5 & sperm associated antigen 5 & 1.947 \\
\hline Gm9743 & predicted gene 9743 & 1.430 \\
\hline$C h k b$ & choline kinase beta & 1.425 \\
\hline Grem2 & gremlin 2 homolog, cysteine knot superfamily & 1.663 \\
\hline Bhlhe41 & basic helix-loop-helix family, mbr. e41 & 1.788 \\
\hline Zfp69 & zinc finger protein 69 & 1.569 \\
\hline Olfr 373 & olfactory receptor 373 & 1.566 \\
\hline Zfp395 & zinc finger protein 395 & 1.409 \\
\hline Apoc1 & apolipoprotein C-I, tr-var. 1 & 1.424 \\
\hline Tspan 2 & tetraspanin 2 & 1.462 \\
\hline 1700047G07Rik & putative uncharacterized protein & 1.576 \\
\hline Chd7 & $\begin{array}{l}\text { chromodomain helicase DNA binding protein } \\
7\end{array}$ & 1.548 \\
\hline Gabarapl2 & GABA(A) receptor-associated protein like 2 & 1.593 \\
\hline
\end{tabular}

\begin{tabular}{|c|c|c|}
\hline Cyp17al & cytochrome P450, fam. 17a, polypeptide 1 & 1.640 \\
\hline $\operatorname{Tb} \times 18$ & T-box 18 & 1.610 \\
\hline Slc15a1 & solute carrier fam. 15 (oligopeptide), mbr. 1 & 1.676 \\
\hline Bex4 & brain expressed gene 4 & 1.427 \\
\hline Epha3 & Eph receptor A3 & 1.442 \\
\hline Itgad & integrin, alpha D & 1.411 \\
\hline $\operatorname{Rgn}$ & regucalcin & 1.568 \\
\hline Olfro & olfactory receptor 9 & 1.477 \\
\hline $\operatorname{Trpc} 7$ & TRP cation channel, subfam.C, mbr. 7 & 1.490 \\
\hline Gm7039 & predicted gene similar to calmodulin. & 1.435 \\
\hline Biccl & bicaudal $\mathrm{C}$ homolog 1 & 1.495 \\
\hline Gpr101 & G protein-coupled receptor 101 & 1.450 \\
\hline Olfr 312 & olfactory receptor 312 & 1.558 \\
\hline$E d a 2 r$ & ectodysplasin $\mathrm{A} 2$ isoform receptor, tr-var. 1 & 1.419 \\
\hline Spatal8 & spermatogenesis associated 18 & 1.472 \\
\hline Gabrb1 & GABA-A receptor, subunit beta 1 & 1.643 \\
\hline Vip & vasoactive intestinal polypeptide & 1.438 \\
\hline V1ra3 & vomeronasal 1 receptor, $\mathrm{A} 3$ & 1.484 \\
\hline Olfr1164 & olfactory receptor 1164 & 1.812 \\
\hline Rai2 & retinoic acid induced 2, tr-var. 1 & 1.616 \\
\hline Lcn 3 & lipocalin 3 & 1.429 \\
\hline Olfr 1459 & olfactory receptor 1459 & 1.618 \\
\hline Spt1 & salivary protein 1 & 1.407 \\
\hline Olfr611 & olfactory receptor 611 & 1.588 \\
\hline A530021J07Rik & putative uncharacterized protein & 1.429 \\
\hline Olfr1166 & olfactory receptor 1166 & 1.482 \\
\hline Gm 10667 & putative uncharacterized protein & 1.661 \\
\hline$H s d 3 b 1$ & $\begin{array}{l}\text { hydroxy- } \Delta \text { - } 5 \text {-steroid dehydrogenase, } \\
3 \beta \text {-steroid- } \Delta \text {-isomerase } 1\end{array}$ & 1.714 \\
\hline Gm5793 & pseudogene & 1.457 \\
\hline Olfr 1255 & olfactory receptor 1255 & 1.513 \\
\hline Mup20 & predicted gene & 1.764 \\
\hline Nlrp $4 c$ & NLR family, pyrin domain containing $4 \mathrm{C}$ & 1.557 \\
\hline Svs $3 b$ & seminal vesicle secretory protein $3 \mathrm{~B}$ & 1.641 \\
\hline $5530400 \mathrm{C} 23 \mathrm{Rik}$ & putative uncharacterized protein & 1.542 \\
\hline Olfr 495 & olfactory receptor 495 & 1.494 \\
\hline Cyp3a57 & cytochrome P450, fam. 3a, polypeptide 57 & 1.490 \\
\hline Olfr 1000 & olfactory receptor 1000 & 1.686 \\
\hline Ssxb10 & synovial sarcoma, $\mathrm{X}$ member $\mathrm{B}$, breakpoint 10 & 1.545 \\
\hline $\operatorname{Vmn} 2 r 78$ & vomeronasal 2 , receptor 78 & 1.742 \\
\hline $\operatorname{V1rc21}$ & vomeronasal 1 receptor, $\mathrm{C} 21$ & 1.419 \\
\hline Olfr 516 & olfactory receptor 516 & 1.636 \\
\hline Zfp 811 & zinc finger protein 811 & 1.413 \\
\hline Сур $2 c 68$ & cytochrome P450, fam. 2c, polypeptide 68 & 1.549 \\
\hline V1rd4 & vomeronasal 1 receptor, D4 & 1.517 \\
\hline LOC100045278 & predicted similar to ribosomal protein S25 & 1.513 \\
\hline$W f d c 6 b$ & WAP four-disulfide core domain $6 \mathrm{~B}$ & 1.467 \\
\hline 9830004L10Rik & adult male bone cDNA & 1.698 \\
\hline Gm 10848 & putative uncharacterized protein & 1.469 \\
\hline
\end{tabular}


Table 2. DNA Microarray Data

\begin{tabular}{|c|c|c|}
\hline Gene & Full name & Ratio \\
\hline \multicolumn{3}{|c|}{ Down-regulated genes } \\
\hline Inmt & indolethylamine $N$-methyltransferase & 0.607 \\
\hline Slcola6 & $\begin{array}{l}\text { solute carrier organic anion transporter fam., } \\
\text { mbr. } 1 \mathrm{a} 6\end{array}$ & 0.581 \\
\hline C730048C13Rik & RIKEN cDNA & 0.306 \\
\hline Tmigdl & $\begin{array}{l}\text { transmemrane \& immunoglobulin domain } \\
\text { containing } 1\end{array}$ & 0.631 \\
\hline Etvl & ets variant gene 1 & 0.588 \\
\hline Stxbp5l & syntaxin binding protein 5 -like, tr-var.xb & 0.633 \\
\hline Aplnr & apelin receptor & 0.632 \\
\hline Sp100 & nuclear antigen Sp100 & 0.615 \\
\hline Asxl3 & isoform 2 of putative polycomb gr. protein & 0.553 \\
\hline Gbp6 & guanylate binding protein 6 & 0.629 \\
\hline Gm4802 & predicted gene & 0.524 \\
\hline $\operatorname{V1rc26}$ & vomeronasal 1 receptor, $\mathrm{C} 26$ & 0.615 \\
\hline Gm11272 & predicted gene & 0.633 \\
\hline Prkar2b & cAMP dependent protein kinase, type II beta & 0.600 \\
\hline Hmcnl & hemicentin 1 & 0.567 \\
\hline Lepr & leptin receptor, tr-var. 3 & 0.613 \\
\hline $\operatorname{Vlrc10}$ & vomeronasal 1 receptor, $\mathrm{C} 10$ & 0.539 \\
\hline F730021E23Rik & B6-derived CD11 + ve dendritic cells cDNA & 0.619 \\
\hline Gm9443 & predicted: similar to LNR42 & 0.611 \\
\hline
\end{tabular}

Abbreviations: gr, group; mbr, member; fam, family; tr-var., transcription variant dep, dependent. epithelium-specific MIF transgenic mice. ${ }^{10)}$ Thus, MIF was screened as a possible candidate for the induction of microalbuminuria in diabetic $d b / d b$ mice by up-regulated transcription in the kidneys and by its own proteinuric properties.

Among the other genes in Table 2, muscle glycogen synthase 1 gene (Gysl) was reported that its polymorphism is related to hypertension and microalbuminuria. ${ }^{11)}$ Fructosebisphosphate aldolase $\mathrm{C}$ gene (Aldoc) was reported that its expression was enhanced together with MIF gene in breast cancer. ${ }^{12)}$ Integrin alpha D (Itgad) was reported to be related to macrophage migration. ${ }^{13)}$ Although these genes may be revealed as further candidate genes for the induction of microalbuminuria, we decided to further investigate the protein profile of MIF in diabetic $d b / d b$ and $o b / o b$ mice.

Urinary excretion of MIF protein increased significantly only in $d b / d b$ mice (Fig. 2A). Moreover, the increase of urinary secretion of MIF was observed significantly from 6-week-old (Fig. 2B). However, the urinary albumin was increased significantly from 8 -week-old, as described in earlier report. Namely, the urinary secretion of MIF preceded microalbuminuria. Consequently, the urinary secretion of MIF is not induced by microalbuminuria, but a possible candidate for the inducer of microalbuminuria in diabetic $d b / d b$ mice. Contrary to the MIF mRNA, MIF protein levels were significantly higher in the kidneys in both $d b / d b$ and $o b / o b$ mice
A.

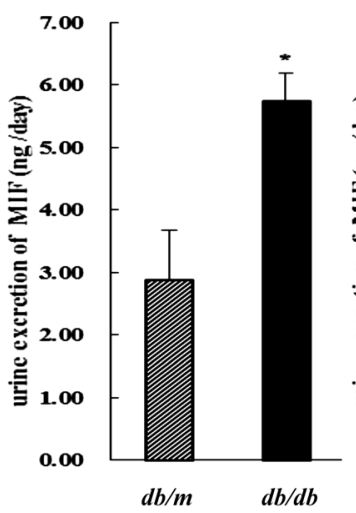

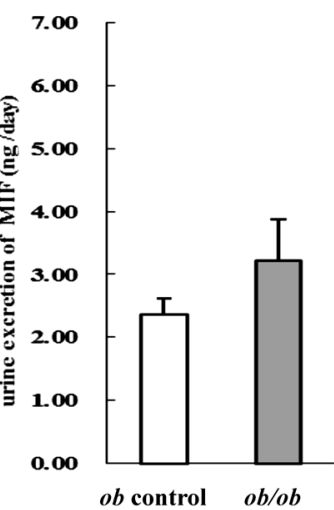

C.
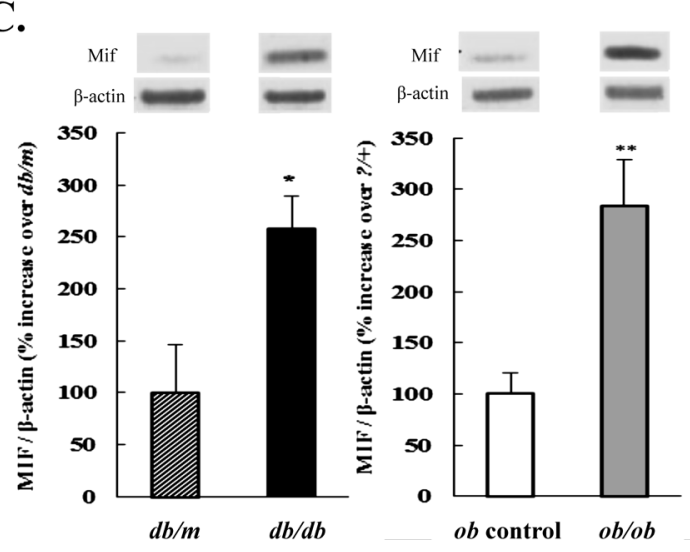

B.

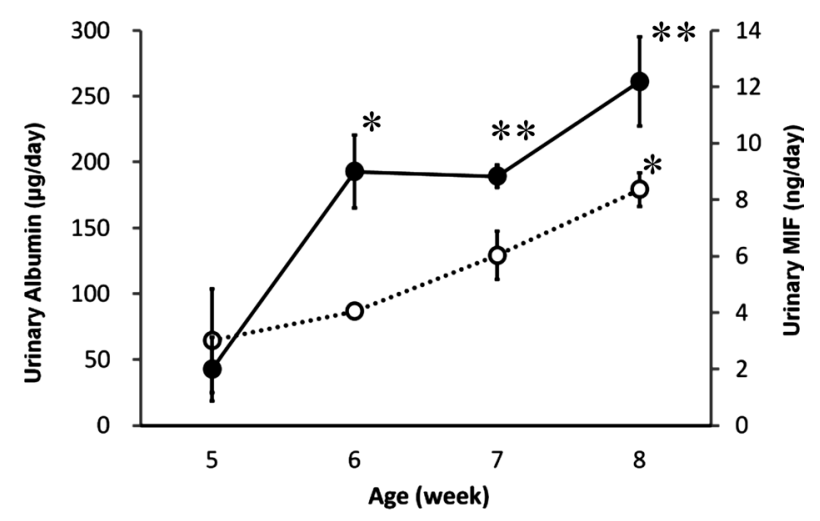

Fig. 2. Expression Profile of MIF Protein

$O b$ control $v s$. $o b / o b$, and the $d b / m v s$. $d b / d b$ strains were compared. Urinary excretion of MIF protein (A) and MIF protein levels in the kidney (C) ( $n=4-6$, mean \pm S.E.M., $* p<0.05, * * p<0.01$ ). The amount of MIF excretion in the urine was elevated in $d b / d b$ mice. Weekly profile of urinary excretion of MIF (closed circle and continuous line) and albumin (open circle and dashed line) in $d b / d b$ mice (B) $(n=3$, mean \pm S.E.M., $* p<0.05, * * p<0.01)$. The urinary excretion of MIF preceded the onset of microalbuminuria. 
A

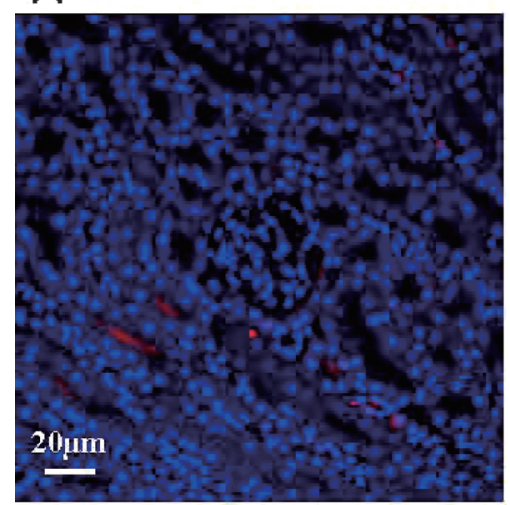

D

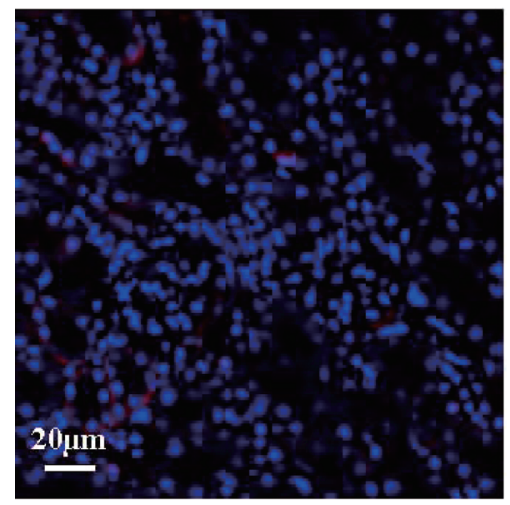

B

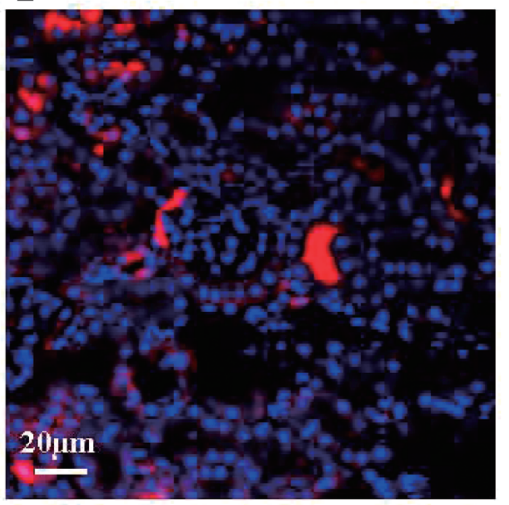

E

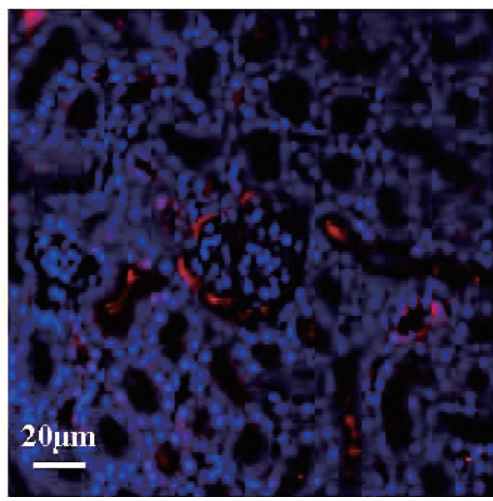

C

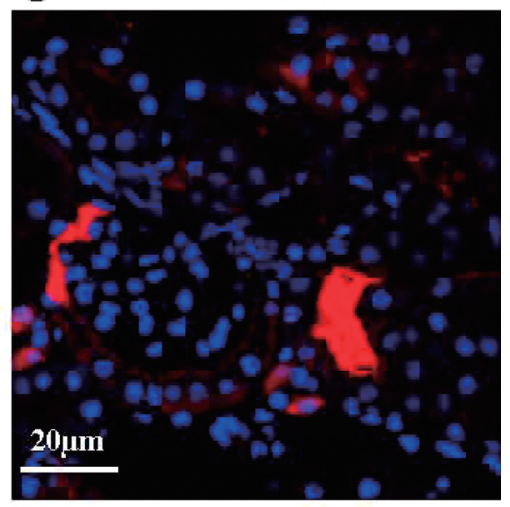

F

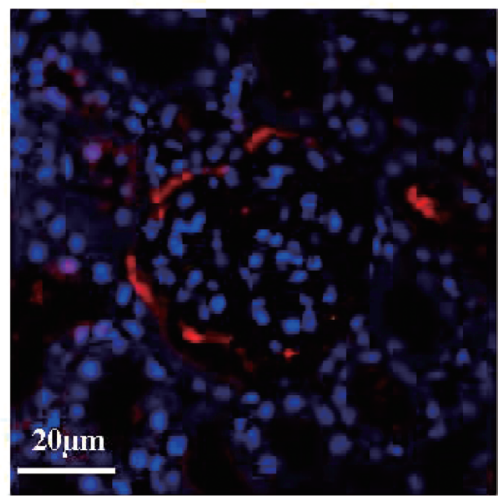

Fig. 3. Localization of MIF Protein in the Kidney

Expression of MIF protein was demonstrated in the tubules or interstitium (A,D) of the kidneys from control mice. MIF appeared to be localized to the Bowman's capsule in the kidneys of $o b / o b$ mice $(\mathrm{B}, \mathrm{C})$ and $d b / d b$ mice $(\mathrm{E}, \mathrm{F})$.

(Fig. 2C). MIF exists in cells in preformed pools and secreted in response to a variety of stimuli including inflammation and hypoxia. ${ }^{14)}$ Consequently, MIF protein was secreted only from the kidneys of $d b / d b$ mice, but was accumulated in the kidneys of both $d b / d b$ and $o b / o b$ mice. Another possible mechanism underlying the observed increase in urinary MIF protein is that MIF protein in the glomerular filtrate may not have been reabsorbed by the tubule and was thus excreted into the urine. However, MIF protein reabsorption failure at the tubule cannot explain the elevated levels of MIF mRNA in the kidneys of $d b / d b$ mice. Consequently, it is possible that the observed increase in urinary MIF protein could be due to secretion of MIF protein from the kidneys of $d b / d b$ mice.

The MIF receptor CD74 is increased in tubular cells and podocyte in human diabetic nephropathy. MIF engagement of CD74 activated mitogen activated protein (MAP) kinase and increased tumor necrosis factor (TNF)-related apoptosisinducing ligand (TRAIL) expression in podocyte, which promote a modest increase in podocyte injury and cell death. ${ }^{15)}$ Podocyte overexpression of MIF caused podocyte injury and proteinuria in mice without macrophage migration. ${ }^{10)}$ Thus, we consider that the observed secretion of urinary MIF protein may be associated with the onset of microalbuminuria in diabetic $d b / d b$ mice. Although it was suggested that in type 2 diabetes macroalbuminuria is the main predictor of mortality, independently of both eGFR and cardiovascular risk factors, ${ }^{16)}$ the spectrum of diabetic nephropathy has recently expanded, as lack of significant albuminuria is present in $30 \%$ of dia- betics with kidney function impairment. ${ }^{17)}$ The MIF-induced TRAIL expression in tubular cell and podocyte also causes renal cell loss and impairment of kidney function. Thus, further study is necessary to clarify the mechanism of secretion of urinary MIF protein observed in diabetic $d b / d b$ mice.

It has already been reported that MIF production in the kidneys of 8-month-old (32-week-old) $d b / d b$ mice invaded by macrophages results in an increase in proinflammatory cytokines, MCP-1 and TNF- $\alpha{ }^{18)}$ In the same report, the invasion of MIF-producing macrophages and an increase in proinflammatory cytokines were not observed in the kidneys of 2-month-old $d b / d b$ mice. Our current study also did not result in the invasion of MIF-producing macrophages in the kidneys of 2-month (8-week)-old $d b / d b$ mice. Rather, MIF was localized to the Bowman's capsule epithelium in the kidneys of both $d b / d b$ and $o b / o b$ mice (Fig. 3), in agreement with previous data concerning normal human kidneys. ${ }^{19)}$ Furthermore, chemokines, which are normally induced by macrophages such as MCP-1 and TNF- $\alpha$, were not elevated in our microarrays. In other words, there was no evidence of proinflammatory cytokine involvement in relation to microalbuminuria in the kidney of early 8 -weeks-old $d b / d b$ mice.

In conclusion, we suggest that the onset of microalbuminuria in early diabetic nephropathy may be associated with the urinary secretion of intra-renal MIF.

Acknowledgements The authors thank Mai Suzuki and Nana Iwakawa for technical assistance. This study was sup- 
ported in part by Grants-in-Aid from the Ministry of Education, Culture, Sports, Science and Technology of Japan (Grant No. 23591340) and from the Gout Research Foundation.

\section{REFERENCES}

1) Akizawa T. Current status of dialysis therapy and related clinical guidelines in Japan. Japan Medical Association Journal, 53, 185-187 (2010)

2) Yokoyama $H$, Okudaira M, Otani $T$, Sato A, Miura J, Takaike $H$, Yamada H, Muto K, Uchigata Y, Ohashi Y, Iwamoto Y. Higher incidence of diabetic nephropathy in type 2 than in type 1 diabetes in early-onset diabetes in Japan. Kidney Int., 58, 302-311 (2000).

3) Sharma K, McCue P, Dunn SR. Diabetic kidney disease in the db/db mouse. Am. J. Physiol. Renal Physiol., 284, F1138-F1144 (2003).

4) Lan HY. Role of macrophage migration inhibition factor in kidney disease. Nephron, Exp. Nephrol., 109, e79-e83 (2008).

5) Hosoyamada M, Takiue Y, Morisaki H, Cheng J, Ikawa M, Okabe M, Morisaki T, Ichida K, Hosoya T, Shibasaki T. Establishment and analysis of SLC22A12 (URAT1) knockout mouse. Nucleosides Nucleotides Nucleic Acids, 29, 314-320 (2010).

6) Takiue Y, Hosoyamada M, Kimura M, Saito H. Enhancement of androgen action in the kidneys of transgenic mice harboring the mutant human UMOD gene. J. Pharmacol. Sci., 115, 383-389 (2011).

7) Takiue Y, Hosoyamada M, Yokoo T, Kimura M, Shibasaki T. Progressive accumulation of intrinsic mouse uromodulin in the kidneys of transgenic mice harboring the mutant human uromodulin gene. Biol. Pharm. Bull., 31, 405-411 (2008).

8) Mizuno TM, Kleopoulos SP, Bergen HT, Roberts JL, Priest CA, Mobbs CV. Hypothalamic pro-opiomelanocortin mRNA is reduced by fasting and [corrected] in $o b / o b$ and $d b / d b$ mice, but is stimulated by leptin. Diabetes, 47, 294-297 (1998).

9) Lan HY, Bacher M, Yang N, Mu W, Nikolic-Paterson DJ, Metz C, Meinhardt A, Bucala R, Atkins RC. The pathogenic role of macrophage migration inhibitory factor in immunologically induced kidney disease in the rat. J. Exp. Med., 185, 1455-1465 (1997).

10) Sasaki S, Nishihira J, Ishibashi T, Yamasaki Y, Obikane $K$, Echigoya M, Sado Y, Ninomiya Y, Kobayashi K. Transgene of MIF induces podocyte injury and progressive mesangial sclerosis in the mouse kidney. Kidney Int., 65, 469-481 (2004).

11) Orho-Melander M, Almgren P, Kanninen T, Forsblom C, Groop LC. A paired-sibling analysis of the $X b a \mathrm{I}$ polymorphism in the muscle glycogen synthase gene. Diabetologia, 42, 1138-1145 (1999).

12) Bini L, Magi B, Marzocchi B, Arcuri F, Tripodi S, Cintorino M, Sanchez JC, Frutiger S, Hughes G, Pallini V, Hochstrasser DF, Tosi P. Protein expression profiles in human breast ductal carcinoma and histologically normal tissue. Electrophoresis, 18, 2832-2841 (1997).

13) Mabon PJ, Weaver LC, Dekaban GA. Inhibition of monocyte/macrophage migration to a spinal cord injury site by an antibody to the integrin alphaD: a potential new anti-inflammatory treatment. Exp. Neurol., 166, 52-64 (2000).

14) Merk M, Baugh J, Zierow S, Leng L, Pal U, Lee SJ, Ebert AD, Mizue Y, Trent JO, Mitchell R, Nickel W, Kavathas PB, Bernhagen J, Bucala R. The Golgi-associated protein p115 mediates the secretion of macrophage migration inhibitory factor. J. Immunol., 182, 6896-6906 (2009).

15) Sanchez-Niño MD, Sanz AB, Ihalmo P, Lassila M, Holthofer H, Mezzano S, Aros C, Groop PH, Saleem MA, Mathieson PW, Langham R, Kretzler M, Nair V, Lemley KV, Nelson RG, Mervaala E, Mattinzoli D, Rastaldi MP, Ruiz-Ortega M, Martin-Ventura JL, Egido J, Ortiz A. The MIF receptor CD74 in diabetic podocyte injury. J. Am. Soc. Nephrol., 20, 353-362 (2009).

16) Bruno G, Merletti F, Bargero G, Novelli G, Melis D, Soddu A, Perotto M, Pagano G, Cavallo-Perin P. Estimated glomerular filtration rate, albuminuria and mortality in type 2 diabetes: the Casale Monferrato study. Diabetologia, 50, 941-948 (2007).

17) Fernández Fernández B, Elewa U, Sánchez-Niño MD, Rojas-Rivera JE, Martin-Cleary C, Egido J, Ortiz A. 2012 update on diabetic kidney disease: the expanding spectrum, novel pathogenic insights and recent clinical trials. Minerva Med., 103, 219-234 (2012).

18) Chow F, Ozols E, Nikolic-Paterson DJ, Atkins RC, Tesch GH. Macrophages in mouse type 2 diabetic nephropathy: correlation with diabetic state and progressive renal injury. Kidney Int., 65, 116-128 (2004).

19) Imamura K, Nishihira J, Suzuki M, Yasuda K, Sasaki S, Kusunoki Y, Tochimaru H, Takekoshi Y. Identification and immunohistochemical localization of macrophage migration inhibitory factor in human kidney. Biochem. Mol. Biol. Int., 40, 1233-1242 (1996). 